\title{
Eficacia y tolerancia de la pentamidina en el tratamiento de la leishmaniasis cutánea producida por Leishmania (V.) panamensis en Colombia
}

\author{
Sara María Robledo, Juan Alberto Puerta, Diana Lorena Muñoz, \\ Mónica Guardo, Iván Darío Vélez \\ Programa de Estudio y Control de Enfermedades Tropicales PECET, Universidad de Antioquia
}

Introducción. El tratamiento de elección de la leishmaniasis cutánea es el antimonio pentavalente con una eficacia cercana al 85\%; sin embargo, debido a los serios efectos adversos y a la aparición cada vez más frecuente de resistencia al tratamiento, se hace necesaria la búsqueda de alternativas terapéuticas.

Objetivo. Evaluar la eficacia terapéutica y la tolerancia del isotianato de pentamidina con un ensayo clínico piloto con pacientes con leishmaniasis cutánea producida por L. (V) panamensis en Colombia.

Materiales y métodos. Se incluyeron en el estudio 63 pacientes los cuales fueron tratados por vía intramuscular con $4 \mathrm{mg}$ de isotianato de pentamidina/kg de peso/ interdiario por cuatro dosis, con seguimiento clínico por seis meses.

Resultados. De los 63 paciente incluidos, 43 fueron evaluados durante los seis meses del estudio y de estos 37 (86\%) presentaron curación de todas sus lesiones mes y medio después de terminar el tratamiento. Cinco pacientes $(11,6 \%)$ fallaron al tratamiento por no cicatrización de las lesiones y uno presentó recaída. En general el tratamiento fue bien tolerado; los efectos adversos principales fueron dolor y edema en el sitio de aplicación, mareo y fiebre, de intensidad leve a moderada. No se presentaron hipoglucemia, hipotensión ni diabetes.

Discusión. Los resultados del presente estudio validan los hallazgos de eficacia de la pentamidina para el tratamiento de la leishmaniasis cutánea en Colombia y en Brasil. Se destaca una baja frecuencia e intensidad de los efectos colaterales en la población civil donde no se encontró ningún paciente con efectos secundarios serios.

Palabra clave: leishmaniasis cutánea, tratamiento, pentamidina, eficacia, tolerancia, Colombia.

Efficacy and tolerance of pentamidine for treatment of cutaneous leishmaniasis caused by por L. (V) panamensis in Colombia

Introduction. Current treatment for human cutaneous leishmaniasis (CL) relies on pentavalent antimonials. Although efficacy of these drugs is high (around $85 \%$ ), their widely documented toxicity and increasing resistance makes the search for therapeutic alternatives a priority for endemic countries.

Objective. To evaluate the efficacy and tolerability of the pentamidine isethionate for the treatment of cutaneous leishmaniasis caused by $L$. (V) panamensis in a pilot clinical trial.

Materials and methods. Sixty three individuals suffering $C L$ were enrolled. Patients received four intramuscular injections of pentamidine ( $4 \mathrm{mg} / \mathrm{Kg} /$ injection) administered every other day, and both clinical efficacy and side effects were documented during the following 6 months.

Results. Of the 63 patients enrolled, 43 could be followed for 6 months. 86\% (37/43) of treated patients healed all their lesions by 1.5 months after therapy. Treatment failure was observed in only five patients $(11.6 \% ; 5 / 43)$. One patient showed relapse. Overall tolerance of treatment was good, with adverse events such as local pain and swelling at the site of injection, dizziness and fever, varying from mild to moderate. Hypoglycemia, hypotension or diabetes were not observed. 
Discussion. These results confirm the previously reported efficacy of pentamidine for the treatment of $\mathrm{CL}$ in Colombia and Brazil, and, additionally, highlight the low intensity and frequency of side effects in a civilian population. No serious adverse events were recorded.

Key words: cutaneous leishmaniasis, therapy, pentamidine, efficacy, tolerance, Colombia.

La leishmaniasis cutánea es una enfermedad endémica en Colombia. Para los años 2003 y 2004 se diagnosticaron anualmente cerca de 10.000 casos y en el 2005 la cifra aumentó a cerca de $18.000,98 \%$ de leishmaniasis cutánea, $0,7 \%$ de leishmaniasis mucosa y $0,9 \%$ de leishmaniasis visceral.

Leishmania (V.) panamensis es la especie más ampliamente extendida en el país, responsable de la mayoría de casos cutáneos y mucosos (1). El tratamiento de elección es el antimonio pentavalente $\left(\mathrm{Sb}^{\vee}\right)$ a dosis de $20 \mathrm{mg} / \mathrm{kg}$ por día, por 20 días con una eficacia cercana al $85 \%$ (2). Sin embargo, debido a la necesidad de aplicar diariamente inyecciones intramusculares (IM), la duración del tratamiento y los efectos colaterales de los antimoniales: mialgias, artralgias, anorexia, náuseas y dolor de cabeza (3), muchos pacientes no concluyen el tratamiento, lo que favorece la reactivación de la lesión, el compromiso en mucosas y la aparición de resistencia al medicamento. Por esta razón, la Organización Mundial de la Salud plantea como prioritaria la búsqueda de alternativas terapéuticas.

El isotianato de pentamidina es un medicamento antiparasitario de uso general utilizado durante muchos años en el tratamiento de infecciones producidas por Trypanosoma, Babesia y Pneumocystis y se ha utilizado como tratamiento alternativo para casos de leishmaniasis visceral resistentes al Sbv $(4,5)$. En Colombia y en otros países, el uso de pentamidina a diferentes dosis como tratamiento alternativo para casos de leishmaniasis cutánea producida por L. (V.) panamensis y Leishmania (V.) braziliensis, ha

\footnotetext{
Correspondencia:

Iván Darío Vélez, Programa de Estudio y Control de Enfermedades Tropicales, PECET, Universidad de Antioquia, Sede de Investigación Universitaria, SIU, Laboratorio 632; Calle 62 № 52-59, Medellín, Colombia.

Teléfono: (574) 210 6502; fax: (574) 2106511

idvelez@udea.edu.co

Recibido: 06/09/05; aceptado: 25/02/06
}

brindado altas tasas de curación, hasta del 96\% (13), con tasas de recaída muy bajas pero con efectos colaterales leves o moderados en esquemas terapéuticos de bajas dosis que incluyen dolor en el sitio de aplicación de la inyección, náuseas, vómito y, en algunos casos, hipoglucemia. Se han observado efectos adversos serios como diabetes mellitus y nefrotoxicidad en esquemas prolongados con altas dosis como los que se emplean en neumonías por Pneumocystis jirovecio en pacientes con coinfección $\mathrm{VIH}$-Leishmania (6-10).

Teniendo en cuenta que los estudios previos realizados en Colombia se llevaron a cabo en población militar y ante la necesidad de validar estos estudios en población general, por iniciativa del Ministerio de Protección Social se diseñó el presente estudio con el fin de evaluar la eficacia y la tolerancia a la pentamidina en población civil con leishmaniasis cutánea, utilizando un esquema terapéutico de $4 \mathrm{mg} / \mathrm{kg}$, interdiario, por cuatro dosis.

\section{Materiales y métodos \\ Diseño del estudio}

Estudio clínico abierto en el que se evaluó la eficacia y la tolerancia de 63 pacientes con diagnóstico parasitológico de leishmaniasis cutánea tratados con isotianato de pentamidina (Pentacarinat ${ }^{\circledR}$ ), suministrado por el Ministerio de Salud, los cuales fueron atendidos por el Programa de Estudio y Control de Enfermedades Tropicales, PECET.

Los criterios de selección para la inclusión de los pacientes fueron: edad, entre 18 y 40 años; diagnóstico parasitológico de leishmaniasis cutánea, sin tratamiento previo para leishmaniasis durante los seis meses anteriores; ausencia de lesiones en mucosas o en regiones cercanas a mucosas; disponibilidad para el seguimiento clínico del tratamiento, y haber aceptado por escrito ser incluido en el estudio. Entre los criterios de 
exclusión se tuvieron en cuenta: embarazo y el padecimiento de alguna enfermedad aguda o crónica asociada o la imposibilidad de asegurar el seguimiento.

Para la realización de este estudio se contó con la aprobación del Comité de Bioética Humana de la Universidad de Antioquia.

Todas las cepas aisladas de los pacientes se identificaron por medio de inmunofluorescencia utilizando los anticuerpos monoclonales gentilmente suministrados por Diane McMahon Pratt.

Los pacientes tratados con pentamidina recibieron una dosis IM de $4 \mathrm{mg} / \mathrm{kg}$ cada dos días por cuatro dosis. Para determinar la respuesta al tratamiento se calculó el área de ulceración e induración de cada una de las lesiones. La evaluación clínica y la medición del área de las lesiones se hizo antes de comenzar el tratamiento, al final del mismo, a las tres y seis semanas y a los seis meses después de terminado el tratamiento.

Para hacer la evaluación de la respuesta al tratamiento se tuvieron en cuenta los siguientes parámetros clínicos, siempre con respecto a la evaluación anterior:

- cicatrización completa: cicatrización del 100\% del área y desaparición completa de la induración;

- mejoría clínica: cicatrización o disminución de la induración mayor al 50\% del área;

- sin mejoría clínica: cicatrización o disminución de la induración menor del $50 \%$ o aumento menor del $50 \%$ del área;

- falla clínica: aumento mayor del 50\% del área;

- recaída: reactivación de alguna lesión después de una cicatrización completa.

Criterio de falla:

1. A las tres semanas: cuando hay, por lo menos, una lesión con induración o ulceración, calificada como falla.

2. A las seis semanas: cuando hay, por lo menos, una lesión con induración o ulceración, calificada como sin mejoría clínica o falla.
3. A los seis meses: cuando todavía hay una lesión con induración o ulceración activa.

4. Durante todo el estudio: cualquier lesión con recaída o cuando hay inicio de una lesión mucosa.

Además, se registraron la aparición y el grado de seriedad de los efectos adversos, clasificados como leves, moderados o graves. Todos los pacientes que presentaron fallas, recibieron tratamiento con antimoniato de meglumina $(20 \mathrm{mg} /$ kg por día, IM, durante 20 días).

\section{Análisis estadístico}

Se realizó un análisis descriptivo de las diferentes variables evaluadas. Para las variables como edad, número de lesiones por paciente y tiempo de evolución de las lesiones, se calcularon el promedio y la desviación estándar y los intervalos de confianza para el promedio de estas variables, utilizando un nivel de confianza del $95 \%$, asumiendo una distribución normal toda vez que el $n$ es mayor de 30 .

\section{Resultados}

Todos los pacientes incluidos en el estudio eran procedentes del noroccidente colombiano y sus características más relevantes se encuentran citadas en el cuadro 1.

De los 63 pacientes incluidos, 43 (68\%) terminaron el seguimiento clínico de los seis meses postratamiento, con los cuales se realizaron los cálculos de eficacia por protocolo. Veinte pacientes se perdieron en el seguimiento y, por lo

Cuadro 1. Características de los pacientes evaluados en el estudio.

\begin{tabular}{|c|c|}
\hline Característica & Valor \\
\hline Pacientes evaluados & 43 \\
\hline Edad en años $(\square \pm D E)^{a}$ & $25,3 \pm 12,5$ \\
\hline Hombres & $74,4 \%$ \\
\hline Lesiones por paciente $(\square \pm D E)^{b}$ & $3,5 \pm 6,3$ \\
\hline Tiempo evolución, meses $(\square \pm D E)^{c}$ & $3,6 \pm 5,7$ \\
\hline Pacientes con cultivo positivo & $39(90,7 \%)$ \\
\hline Leishmania panamensis & $39(100 \%)$ \\
\hline
\end{tabular}

Intervalos de confianza del 95\%: a 21,57-29,03; b 1,62-5,38; c1,9-5,3 
Cuadro 2. Eficacia del tratamiento con pentamidina.

\begin{tabular}{|c|c|c|}
\hline Variable & $\begin{array}{l}\text { Intención } \\
\text { por tratar }\end{array}$ & $\begin{array}{c}\text { Protocolo } \\
n\end{array}$ \\
\hline Pacientes incluidos & 63 & 43 \\
\hline $\begin{array}{l}\text { Cicatrización completa a } \\
\text { la semana } 3\end{array}$ & $28,6 \%$ & $41,9 \%$ \\
\hline $\begin{array}{l}\text { Cicatrización completa a } \\
\text { la semana } 6\end{array}$ & $60,3 \%$ & $88,3 \%$ \\
\hline Recaída a los 6 meses & $1,6 \%$ & $2,3 \%$ \\
\hline Eficacia a los 6 meses & $58,7 \%$ & $86 \%$ \\
\hline
\end{tabular}

tanto, no se obtuvieron datos de su evolución final después del tratamiento y se analizan en el cuadro 2 como intención por tratar (cuadro 2).

Treinta y nueve de los 43 pacientes analizados por protocolo (91\%) tuvieron cultivo positivo. El $100 \%$ de las cepas identificadas con anticuerpos monoclonales correspondieron a $L$. (V.) panamensis.

Se observó completa cicatrización de todas las lesiones en 37 (86\%) de los pacientes evaluados por protocolo, de los cuales $18(41,9 \%)$ cicatrizaron a las tres semanas postratamiento y 19 (44,2\%) a las seis semanas. Cinco (11,6\%) pacientes presentaron falla durante el tiempo de seguimiento. Un paciente que había cicatrizado presentó recaída en la evaluación de los seis meses postratamiento. En ningún paciente se observó aparición de compromiso mucoso. La tasa de eficacia acumulada para el tratamiento fue de $86 \%$, eficacia que disminuye a $58,7 \%$ en el análisis por intención por tratar, si se asume que todos los pacientes perdidos en el seguimiento a los seis meses hubieran fallado al tratamiento.

El $55,8 \%$ de los pacientes tratados con isotianato de pentamidina presentaron efectos secundarios leves o moderados, a saber: dolor en el sitio de aplicación (34,8\%), edema en el sitio de aplicación $(11,6 \%)$, mareos $(11,6 \%)$, fiebre $(9,3 \%)$, cefalea $(4,6 \%)$, adinamia $(4,6 \%)$, náuseas $(2,3 \%)$ y dolor articular $(2,3 \%)$, que fueron evaluados como leves o moderados (figura 1). No se presentaron casos de efectos adversos serios, hipotensión, diabetes mellitus o hipoglucemia.

\section{Discusión}

El isotianato de pentamidina a una dosis de $4 \mathrm{mg} /$ $\mathrm{kg}$, cada dos días, por cuatro dosis, demostró ser efectivo en el $86 \%$ efectivo para el tratamiento en población general colombiana de la leishmaniasis cutánea producida por $L$. (V.) panamensis, proporcionando curación clínica con una baja tasa de recaída $(2,3 \%)$, cuando el seguimiento se hace durante seis meses.

Estos resultados son comparables con los encontrados por Soto et al. (6) quienes evaluaron la aplicación de $2 \mathrm{mg}$ de isotianato de pentamidina/ $\mathrm{kg}$, cada dos días por siete dosis y encontraron una tasa de curación del $96 \%$. Sin embargo, 17,4\% de los pacientes interrumpieron el tratamiento luego de cuatro a seis inyecciones por problemas de hipotensión $(8,7 \%)$, hipoglucemia $(8,7 \%)$, intenso dolor de cabeza $(4,3 \%)$ y mialgias $(4,3 \%)$. Cuando el número de dosis fue de 4 , la eficacia disminuyó al 74\%.

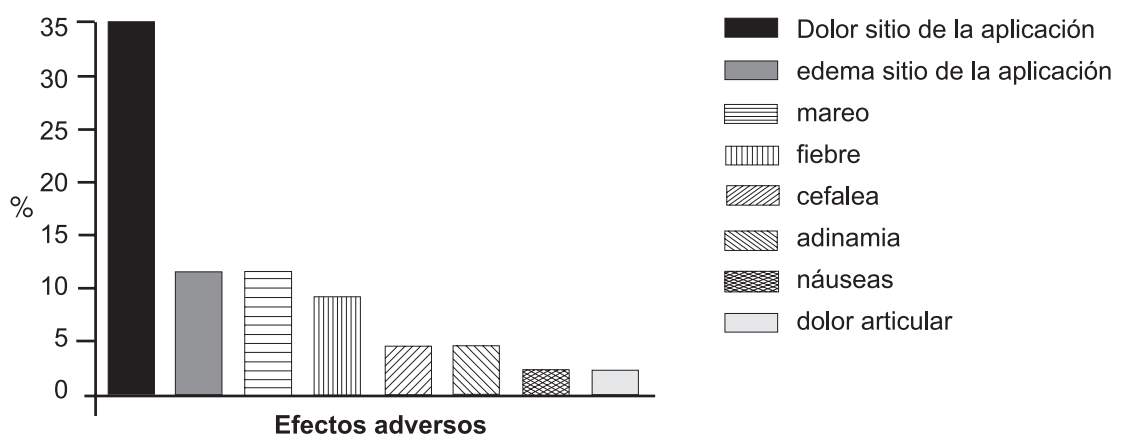

Figura 1. Tolerancia al tratamiento (porcentaje de aparición de los efectos adversos) 
El mismo autor en un estudio posterior en 38 militares colombianos (7), utilizó como esquema de tratamiento $2 \mathrm{mg} / \mathrm{kg}$ cada dos días por cuatro inyecciones y la tasa de curación fue de $84 \%$ pero con una alta frecuencia de efectos adversos (55\%). Si la dosis diaria se aumentaba a $3 \mathrm{mg} /$ $\mathrm{kg}$, la tasa de curación era de $96 \%$ pero el $64 \%$ de los pacientes presentaron manifestaciones secundarias que fueron clasificadas en 10,9\% como moderadas o serias. Con resultados similares, de Paula et al. (11) en Brasil evaluaron la pentamidina en pacientes con lesihmaniosis cutánea producida por Leishmania braziliensis a la dosis de $4 \mathrm{mg} / \mathrm{kg}$ por dosis, interdiarios, por tres dosis y encontraron una eficacia de $71 \%$. Todos estos resultados difieren de los encontrados por Andersen et al. (12) en Perú quienes trataron con isotianato de pentamidina a la dosis de $2 \mathrm{mg} /$ $\mathrm{kg}$, interdiario, por siete inyecciones a 40 pacientes peruanos con leishmaniasis cutánea debida a $L$. braziliensis, con una tasa de curación de sólo el $35 \%$.

En el presente estudio se encontró una tolerancia aceptable al medicamento, la intensidad de los efectos adversos fue menor que la encontrada por Soto en población militar y ningún paciente presentó efectos adversos serios.

Luego de terminado el presente estudio, se informaron algunos casos de rabdomiólisis asintomática y transitoria en pacientes con leishmaniasis cutánea producida por Leishmania guyanensis y que fueron tratados con isotianato de pentamidina en dosis única de $7 \mathrm{mg} / \mathrm{kg}$. Estos pacientes presentaron aumento de la creatíncinasa, sin cambios en los niveles de urea o creatinina, o aumento de creatinín-fosfocinasa y de mioglobina. Es recomendable, por lo tanto, controlar los niveles séricos de proteina cinasa $\mathrm{C}$, aldolasas, mioglobina y transaminasas (ASAT y ALAT) a fin de evitar la aparición de rabdomiólisis o de posibles efectos nefrotóxicos de este medicamento (13-17).

En un ensayo clínico realizado en el PECET con 66 pacientes con diagnóstico parasitológico de leishmaniosis cutánea procedentes de la misma región geográfica de los incluidos en el presente estudio, se evaluó el antimoniato de meglumina a la dosis de $20 \mathrm{mg} \mathrm{Sb} / \mathrm{kg}$ por día durante 20 días y se encontró una eficacia del $93 \%$ (3).

Se concluye que el isotianato de pentamidina y el antimoniato de meglumina son medicamentos comparables en cuanto a su efectividad y a su vía de aplicación. La ventaja del isotianato de pentamidina radica en un menor tiempo de administración y de dosis requerida. La desventaja radica en su costo superior al de los antimoniales, por lo que se debe mantener como un medicamento alternativo para el tratamiento de la leishmaniosis cutánea en Colombia, con el esquema de $4 \mathrm{mg} /$ kg por dosis, interdiario, por cuatro dosis.

\section{Agradecimientos}

Los autores agradecen a los pacientes por su participación voluntaria en el estudio y al Ministerio de Protección Social de Colombia por la invitación a realizar el presente estudio clínico.

\section{Conflicto de interés}

Los autores manifiestan no tener ningún conflicto de interés con la realización de este trabajo.

\section{Financiación}

Este estudio fue financiado por el Programa de Estudio y Control de Enfermedades Tropicales, PECET, Universidad de Antioquia, y por el Ministerio de la Protección Social de Colombia que suministró los medicamentos.

\section{Referencias}

1. Osorio LE, Castillo CM, Ochoa MT. Mucosal leishmaniasis due to Leishmania (Viannia) panamensis in Colombia: clinical characteristics. Am J Trop Med Hyg 1998;59:49-52.

2. Chan-Bacab MJ, Peña Rodríguez LM. Plant natural products with leishmanicidal activity. Nat Prod Rep 2001;18:674-88.

3. Vélez ID, Agudelo S, Hendrickx E, Puerta J, Grogl M, Modabber $\mathbf{F}$ et al. Inefficacy of allopurinol as monotherapy for Colombian cutaneous leishmaniasis. A randomized controlled trial. Ann Inter Med 1997;126:232-6.

4. Thakur CP, Kumar M, Pandey AK. Comparison of regimes of treatment of antimony-resistant kala-azar patients: a randomized study. Am J Trop Med Hyg 1991;45:435-41.

5. World Health Organization. Division of Control of Tropical Diseases. Leishmaniasis control home page. 
[Consultado: 16 de noviembre de 2005]. Disponible en: www.who.int/health-topics/leishmaniasis.htm.

6. Soto-Mancipe J, Grogl M, Berman JD. Evaluation of pentamidine for the treatment of cutaneous leishmaniasis in Colombia. Clin Infect Dis 1993;16:41725.

7. Soto J, Buffet P, Grogl M, Berman J. Successful treatment of Colombian cutaneous leishmaniasis with four injections of pentamidine. Am J Trop Med Hyg 1994;50:107-11.

8. Amato VS, De Paula JG, Imamura R, Amato Neto V, Duarte MI, Boulos $\mathrm{M}$ et al. Treatment of American cutaneous leishmaniasis, with lesions in the mucosa, using pentamidine isethionate. Rev Soc Bras Med Trop 1996;29:477-81.

9. Correia D, Macedo VO, Carvalho EM, Barral A, Magalhaes AV, de Abreu MV et al. Comparative study of meglumine antimoniate, pentamidine isethionate and aminosidine sulfate in the treatment of primary skin lesions caused by Leishmania (Viannia) braziliensis. Rev Soc Bras Med Trop 1996; 29:447-53.

10. Amato V, Amato J, Nicodemo A, Uip D, AmatoNeto V, Duarte M. Treatment of mucocutaneous leishmaniasis with pentamidine isehionate. Ann Dermatol Venereol 1998;125:492-5.

11. de Paula CD, Sampaio JH, Cardoso DR, Sampaio RN. A comparative study between the efficacy of pentamidine isothionate given in three doses for one week and N-methil- glucamine in a dose of $20 \mathrm{mgSbV} /$ day for 20 days to treat cutaneous leishmaniasis. Rev Soc Bras Med Trop 2003;36:365-71.

12. Andersen EM, Cruz-Saldarriaga M, Llanos-Cuentas A, Luz-Cjuno M, Echevarria J, Miranda-Verastegui C et al. Comparison of meglumine antimoniate and pentamidine for Peruvian cutaneous leishmaniasis. Am J Trop Med Hyg 2005;72:133-7.

13. Singh S, Sivakumar R. Challenges and new discoveries in the treatment of leishmaniasis. J Infect Chemother 2004:10:307-15.

14. Lieber-Mbomeyo A, Lipsker D, Milea M, Heid E. Rhabdomyolysis induced by pentamidine (Pentacarinat) during treatment of cutaneous leishmaniasis: 2 cases. Ann Dermatol Venereol 2002;129:50-2

15. Lightburn E, Morand JJ, Meynard JB, Kraemer P, Chaudier B, Pages F et al. Management of American cutaneous leishmaniasis. Outcome apropos of 326 cases treated with high-dose pentamidine isethionate. Med Trop 2003;63:35-44.

16. Delobel P, Pradinaud R. Rhabdomyolysis associated with pentamidine isethionate therapy for American cutaneous leishmaniasis. J Antimicrob Chemother 2003;51:1319-20.

17. Hauben M, Reich L. A case report of rhabdomyolysis with pentamidine that prompted a retrospective evaluation of a pharmacovigilance tool under investigation. Br J Clin Pharmacol 2004;58:675-6. 